\title{
Erratum to: Rock Dynamic Fracture Toughness Tested with Holed-Cracked Flattened Brazilian Discs Diametrically Impacted by SHPB and its Size Effect
}

\author{
Q.Z. Wang $\cdot$ S. Zhang $\cdot$ H.P. Xie
}

Published online: 18 September 2009

(C) Society for Experimental Mechanics 2009

Erratum to: Experimental Mechanics

DOI 10.1007/s11340-009-9265-2

In the above mentioned article, the acknowledgment is missing. It should read as follows:

Acknowledgment This work was supported by the National Natural Science Foundation of China (project no. 10472075).

The online version of the original article can be found under http://dx. doi.org/10.1007/s11340-009-9265-2.

Q.Z. Wang $\cdot$ S. Zhang $\cdot$ H.P. Xie

Department of Civil Engineering and Applied Mechanics,

Sichuan University,

Chengdu, Sichuan 610065, China

Q.Z. Wang $(\bowtie)$

State Key Laboratory of Hydraulics and Mountain

River Engineering,

Chengdu, Sichuan 610065, China

e-mail: qzwang2004@163.com

\section{S. Zhang}

Department of Mining Engineering,

Henan Polytechnic University,

Jiaozhuo, Henan 454001, China 